${ }^{1}$ Sośnicowice Branch Institute of Plant Protection - National Research Institute

${ }^{2}$ Regional Experimental Station in Rzeszów

Institute of Plant Protection - National Research Institute

\title{
THE EFFECTS OF CHEMICAL CONTROL USED AGAINST MALES OF DIABROTICA VIRGIFERA LeCONTE IN MAIZE IN SOUTHERN POLAND IN 2011-2012*
}

\author{
EFEKTY CHEMICZNEGO ZWALCZANIA \\ SAMCÓW DIABROTICA VIRGIFERA LeCONTE \\ NA KUKURYDZY W POŁUDNIOWEJ POLSCE W LATACH 2011-2012
}

\begin{abstract}
Summary. The study was carried out in 2011-2012 in Sławików, southern Poland, on a maize crop monoculture. Diabrotica virgifera males were controlled with insecticides containing active ingredients: acetamiprid, dimethoate, indoxacarb, lambda-cyhalothrin, methaflumizon, thiacloprid and thiacloprid with deltamethrin. The optimum dates of beetle control were established by using PAL pheromone traps from Csalomon ${ }^{\circledR}$ company. Plants were sprayed once or twice with the investigated insecticides during the peak of beetle population in the second half of August. All investigated active ingredients effectively reduced the pest population, usually within 7 days of plant spraying. The second chemical treatment effectively reduced the pest population for a further 7 days.
\end{abstract}

Key words: western corn rootworm, beetles, maize, insecticides, active ingredient

\section{Introduction}

Western corn rootworm (WCR) (Diabrotica virgifera LeConte) is one of the major maize pests in North America. In the USA loss in maize yield caused by WCR together with expenses for its control are estimated at about one milliard dollars a year (Metcalf,

${ }^{*}$ The study was carried out under the Multiannual Programme of the Institute of Plant Protection - NRI, for 2011-2015, Measure 2.2. "Pest risk analysis and management programmes for western corn rootworm (Diabrotica virgifera LeConte)". 
Drzewiecki, S., Pietryga, J., Bereś, P. K. (2016). The effects of chemical control used against males of Diabrotica virgifera LeConte in maize in southern Poland in 2011-2012. Nauka Przyr. Technol., 10, 1, \#7. DOI: 10.17306/J. NPT.2016.1.7

1986). Diabrotica virgifera was brought accidentally to Europe from North America and for the first time officially detected in 1992 in Serbia (Bača, 1994). Ciosi et al. (2008) reported that WCR was introduced into Europe independently in several places in the 1990s and in the early 21 st century, e.g. in Italy, France, the United Kingdom, and this was confirmed by findings on the genetic structure of D. virgifera individuals. WCR spread further across Europe, and by the end of 2011 it had been detected in 22 European countries (EPPO, 2012). In Poland, WCR occurred for the first time in the south-east in 2005 (Sahajdak et al., 2006), and has been continually detected to the present day in almost half of the area of the country.

Since early 2014 WCR has been under compulsory monitoring in individual member states due to its status as a quarantine pest in the European Union (Decyzja..., 2014; Rozporządzenie..., 2014). One of the aims of the monitoring was to identify optimum dates for the chemical control of beetles (Kiss et al., 2005). Chemical treatments reduce the number of fertilized adult females, which, by depositing up to 1400 eggs, give a start to a new, larger generation of the pest (Bereś and Sionek, 2010a; Ciobanu et al., 2011/A; van Rozen and Ester, 2010).

The control of beetles in maize crop monocultures can reduce the population of larvae in the next growing season, and thus limit the loss in yield caused by this pest (Meinke, 1995; Pruess et al., 1974). Moreover, chemical control limits the dispersal of beetles to new sites by preventing long-distance flights and reducing the risk of their transport in vehicles and plant material (Bača, 1994; Baufeld and Enzian, 2005).

In some countries decisions on the chemical control of WCR beetles are made based on the economic injury levels which are compliant with the principles of integrated pest management specified in the directive of the European Parliament and of the Council 2009/128/EC (Dyrektywa..., 2009). In Poland no levels of economic injury have been established for WCR. According to Sivčev et al. (1994), in Serbia the economic injury levels are 10 beetles per plant for commercially grown maize varieties and five beetles per plant for seed maize. On the other hand, Tuska et al. (2002) reported that the presence of one-three beetles per maize cob at the stage of shooting tassels justifies pest control, because their feeding in this period may cause up to $13 \%$ losses in grain yield.

Van Rozen and Ester (2010) reported a wide range of insecticides for the control of WCR beetles but their choice differs by country. In Poland there are currently four insecticides approved for the control of adult forms of $D$. virgifera, and they contain either indoxacarb (three products) or thiacloprid + deltamethrin (one product).

The aim of the carried out study was to assess the efficiency of single or double spraying of maize plants with insecticides containing different active ingredients in the control of WCR beetles.

\section{Materials and methods}

The study was carried out in 2011-2012 in Sławików, Silesian province (southern Poland), on a maize crop monoculture. Observations were carried out on a medium-early maize variety 'Amadeo' (maturity class FAO 230). The experiment was established based on authorization no. WF-411d/2/2010 from the Main Inspectorate of Plant Health and Seed Inspection. 
Drzewiecki, S., Pietryga, J., Bereś, P. K. (2016). The effects of chemical control used against males of Diabrotica virgifera LeConte in maize in southern Poland in 2011-2012. Nauka Przyr. Technol., 10, 1, \#7. DOI: 10.17306/J. NPT.2016.1.7

Maize was sown on 28 April (2011) and 30 April (2012), at a seeding rate of 90000 seeds per 1 ha. The experiment was designed as a system of completely randomized blocks in four replicates. Each plot had a surface area of $50 \mathrm{~m}^{2}$. Each plot's shape was close to a square and measured $6.0 \times 8.3 \mathrm{~m}$ (width $\times$ length). Buffer zones set up between the plots were $3.0 \mathrm{~m}$ wide along the longer side and $2.5 \mathrm{~m}$ wide along the shorter side of each plot. Seven insecticides, listed in Table 1, containing different active ingre-

Table 1. Insecticides used in the experiment, their doses and application dates

Tabela 1. Insektycydy użyte w doświadczeniu, ich dawki oraz terminy zastosowania

\begin{tabular}{|c|c|c|c|}
\hline $\begin{array}{l}\text { No. } \\
\text { Lp. }\end{array}$ & $\begin{array}{l}\text { Application date, experimental setup } \\
\text { Termin zastosowania, kombinacja doświadczalna }\end{array}$ & $\begin{array}{l}\text { Active ingredient } \\
\text { Substancja czynna }\end{array}$ & $\begin{array}{l}\text { Dose per } 1 \text { ha } \\
\text { Dawka na } 1 \text { ha }\end{array}$ \\
\hline 1. & Control - Kontrola & - & - \\
\hline 2. & T1: Proteus 110 OD & Thiacloprid + deltamethrin & 0.751 \\
\hline 3. & T1: Steward 30 WG & Indoxacarb & $0.15 \mathrm{~kg}$ \\
\hline 4. & T1: Alverde $240 \mathrm{SC}$ & Methaflumizon & 0.251 \\
\hline 5. & T1: Mospilan 20 SP & Acetamiprid & $0.08 \mathrm{~kg}$ \\
\hline 6. & T1: Karate Zeon 050 CS & Lambda-cyhalothrin & 0.21 \\
\hline 7. & $\begin{array}{l}\text { T1: Alverde } 240 \text { SC } \\
\text { T2: Karate Zeon 050 CS }\end{array}$ & $\begin{array}{l}\text { Methaflumizon } \\
\text { Lambda-cyhalothrin }\end{array}$ & $\begin{array}{l}0.251 \\
0.21\end{array}$ \\
\hline 8. & $\begin{array}{l}\text { T1: Alverde } 240 \mathrm{SC} \\
\text { T2: Steward } 30 \mathrm{WG}\end{array}$ & $\begin{array}{l}\text { Methaflumizon } \\
\text { Indoxacarb }\end{array}$ & $\begin{array}{l}0.251 \\
0.15 \mathrm{~kg}\end{array}$ \\
\hline 9. & $\begin{array}{l}\text { T1: Proteus } 110 \text { OD } \\
\text { T2: Karate Zeon 050 CS }\end{array}$ & $\begin{array}{l}\text { Thiacloprid + deltamethrin } \\
\text { Lambda-cyhalothrin }\end{array}$ & $\begin{array}{l}0.751 \\
0.21\end{array}$ \\
\hline 10. & $\begin{array}{l}\text { T1: Proteus } 110 \text { OD } \\
\text { T2: Alverde } 240 \mathrm{SC}\end{array}$ & $\begin{array}{l}\text { Thiacloprid + deltamethrin } \\
\text { Methaflumizon }\end{array}$ & $\begin{array}{l}0.751 \\
0.251\end{array}$ \\
\hline 11. & $\begin{array}{l}\text { T1: Mospilan } 20 \mathrm{SP} \\
\text { T2: Alverde } 240 \mathrm{SC}\end{array}$ & $\begin{array}{l}\text { Acetamiprid } \\
\text { Methaflumizon }\end{array}$ & $\begin{array}{l}0.08 \mathrm{~kg} \\
0.25 \mathrm{l}\end{array}$ \\
\hline 12. & $\begin{array}{l}\text { T1: Mospilan } 20 \text { SP } \\
\text { T2: Bi } 58 \text { Nowy } 400 \text { EC }\end{array}$ & $\begin{array}{l}\text { Acetamiprid } \\
\text { Dimethoate }\end{array}$ & $\begin{array}{l}0.08 \mathrm{~kg} \\
0.51\end{array}$ \\
\hline 13. & $\begin{array}{l}\text { T1: Steward } 30 \mathrm{WG} \\
\text { T2: Mospilan } 20 \mathrm{SP}\end{array}$ & $\begin{array}{l}\text { Indoxacarb } \\
\text { Acetamiprid }\end{array}$ & $\begin{array}{l}0.15 \mathrm{~kg} \\
0.08 \mathrm{~kg}\end{array}$ \\
\hline 14. & $\begin{array}{l}\text { T1: Steward } 30 \mathrm{WG} \\
\text { T2: Calypso } 480 \mathrm{SC}\end{array}$ & $\begin{array}{l}\text { Indoxacarb } \\
\text { Thiacloprid }\end{array}$ & $\begin{array}{l}0.15 \mathrm{~kg} \\
0.21\end{array}$ \\
\hline 15. & $\begin{array}{l}\text { T1: Steward } 30 \mathrm{WG} \\
\text { T2: Proteus } 110 \mathrm{OD}\end{array}$ & $\begin{array}{l}\text { Indoxacarb } \\
\text { Thiacloprid + deltamethrin }\end{array}$ & $\begin{array}{l}0.15 \mathrm{~kg} \\
0.75 \mathrm{l}\end{array}$ \\
\hline 16. & $\begin{array}{l}\text { T1: Karate Zeon } 050 \text { CS } \\
\text { T2: Mospilan } 20 \mathrm{SP}\end{array}$ & $\begin{array}{l}\text { Lambda-cyhalothrin } \\
\text { Acetamiprid }\end{array}$ & $\begin{array}{l}0.21 \\
0.08 \mathrm{~kg}\end{array}$ \\
\hline 17. & $\begin{array}{l}\text { T1: Bi } 58 \text { Nowy } 400 \text { EC } \\
\text { T2: Karate Zeon } 050 \text { CS }\end{array}$ & $\begin{array}{l}\text { Dimethoate } \\
\text { Lambda-cyhalothrin }\end{array}$ & $\begin{array}{l}0.51 \\
0.21\end{array}$ \\
\hline 18. & $\begin{array}{l}\text { T1: Calypso } 480 \text { SC } \\
\text { T2: Bi } 58 \text { Nowy } 400 \text { EC }\end{array}$ & $\begin{array}{l}\text { Thiacloprid } \\
\text { Dimethoate }\end{array}$ & $\begin{array}{l}0.21 \\
0.51\end{array}$ \\
\hline
\end{tabular}


Drzewiecki, S., Pietryga, J., Bereś, P. K. (2016). The effects of chemical control used against males of Diabrotica virgifera LeConte in maize in southern Poland in 2011-2012. Nauka Przyr. Technol., 10, 1, \#7. DOI: 10.17306/J. NPT.2016.1.7

dients, were used in the experiment. There were five treatments which were sprayed once (at T1 date) and 12 treatments sprayed twice (at T1 and T2 dates).

Dates of maize spraying were established based on the observations of the number of $D$. virgifera males in PAL pheromone traps from Csalomon ${ }^{\circledR}$ company. In the study years the optimum dates of beetle control were:

- T1: 16 August in 2011 and 20 August in 2012, when maize was at the stage BBCH 71-73 (2011) and BBCH 75-79 (2012) (Adamczewski and Matysiak, 2011),

- T2: 23 August in 2011 and 28 August in 2012, when maize was at the stage BBCH 73-83.

A pheromone trap was placed in the middle of each plot to determine the number of insects and the efficiency of treatments. Counts of male beetles were assessed directly prior to each application and performed seven more times every 3-7 days. The last assessment was conducted 14 days after the T2 treatment. Each time, the beetles were counted and removed from the trap.

Insecticides were applied using an experimental pressure sprayer (type WACH-3) with a boom and TEEJet XR 11003 nozzles, at water consumption of 400 1/ha.

One-way analysis of variance ANOVA in a random block design was used for the statistical analysis of results. The significance of differences between means was analysed by using Tukey's test at $\mathrm{p}<0.05$ and STATISTICA software, version 8.0. Means for homogeneous groups presented in tables are identified with the same letters.

\section{Results}

In 2011-2012, comparable distribution of mean air temperatures was recorded during the beginning of flights and dynamic migrations of WCR beetles (July-August). In 2011 the highest air temperature was recorded in the second ten days of July and the last 10 days of August. The first 10 days of July was the warmest period in 2012. In both growing seasons of the study the total precipitation was greater in July (132-119 mm) than in August (60.9-53.2 mm). Changes in weather conditions during the study period are presented in Table 2.

In 2011-2012 the infestation of the maize grown in two- and three-year long monocultures by $D$. virgifera beetles was variable. Directly before chemical control of the pest the mean number of beetles captured in traps places on experimental fields was 22.3-36.8 in 2011 and 7.5-12.5 in 2012. The number of beetles was higher in 2011, which was mainly attributed to more favourable weather conditions (Table 3 and 4).

In 2011 the population peak of $D$. virgifera beetles on the maize field was observed in the second half of August. The first chemical treatment (T1) was carried out on 16 August, when plants were at developmental stage BBCH 71-73. On the first day after treatment $\mathrm{T} 1$ the best insecticidal effect was noted for products containing lambda-cyhalothrin, thiacloprid + deltamethrin, dimethoate and methaflumizon. These active ingredients were highly effective in controlling WCR beetles up to day 7 after treatment $\mathrm{T} 1$ and significantly reduced the number of insects found in traps (4.5-12.8 beetles per trap) (Table 3). 
Drzewiecki, S., Pietryga, J., Bereś, P. K. (2016). The effects of chemical control used against males of Diabrotica virgifera LeConte in maize in southern Poland in 2011-2012. Nauka Przyr. Technol., 10, 1, \#7. DOI: 10.17306/J. NPT.2016.1.7

Table 2. Weather conditions in Sławików in 2011-2012

Tabela 2. Warunki pogodowe w miejscowości Sławików w latach 2011-2012

\begin{tabular}{|c|c|c|c|c|c|c|c|}
\hline \multirow{2}{*}{$\begin{array}{l}\text { Year } \\
\text { Rok }\end{array}$} & \multirow{2}{*}{$\begin{array}{l}\text { Month } \\
\text { Miesiąc }\end{array}$} & \multirow{2}{*}{$\begin{array}{l}\text { Weather parameters } \\
\text { Parametry meteorologiczne }\end{array}$} & \multicolumn{3}{|c|}{ Decade - Dekada } & \multirow{2}{*}{$\begin{array}{l}\text { Mean } \\
\text { Srednia }\end{array}$} & \multirow{2}{*}{$\begin{array}{l}\text { Monthly } \\
\text { sum } \\
\text { Mie- } \\
\text { sięczna } \\
\text { suma }\end{array}$} \\
\hline & & & I & II & III & & \\
\hline \multirow[t]{10}{*}{2011} & \multirow[t]{2}{*}{$\begin{array}{l}\text { April } \\
\text { Kwiecień }\end{array}$} & $\begin{array}{l}\text { Daily average air temperature }\left({ }^{\circ} \mathrm{C}\right) \\
\text { Średnia dobowa temperatura powietrza }\left({ }^{\circ} \mathrm{C}\right)\end{array}$ & 10.6 & 8.5 & 14.3 & 11.1 & \\
\hline & & $\begin{array}{l}\text { Rainfall sum }(\mathrm{mm}) \\
\text { Suma opadów }(\mathrm{mm})\end{array}$ & 17.4 & 30.1 & 5.5 & & 53.0 \\
\hline & \multirow[t]{2}{*}{$\begin{array}{l}\text { May } \\
\text { Maj }\end{array}$} & \begin{tabular}{|l|} 
Daily average air temperature $\left({ }^{\circ} \mathrm{C}\right)$ \\
Średnia dobowa temperatura powietrza $\left({ }^{\circ} \mathrm{C}\right)$
\end{tabular} & 9.8 & 15.5 & 17.6 & 14.3 & \\
\hline & & $\begin{array}{l}\text { Rainfall sum }(\mathrm{mm}) \\
\text { Suma opadów }(\mathrm{mm})\end{array}$ & 34.4 & 5.5 & 16.2 & & 56.1 \\
\hline & \multirow[t]{2}{*}{$\begin{array}{l}\text { June } \\
\text { Czerwiec }\end{array}$} & $\begin{array}{l}\text { Daily average air temperature }\left({ }^{\circ} \mathrm{C}\right) \\
\text { Średnia dobowa temperatura powietrza }\left({ }^{\circ} \mathrm{C}\right)\end{array}$ & 19.5 & 18.6 & 18.3 & 18.8 & \\
\hline & & $\begin{array}{l}\text { Rainfall sum }(\mathrm{mm}) \\
\text { Suma opadów }(\mathrm{mm})\end{array}$ & 0.2 & 0.0 & 64.1 & & 64.3 \\
\hline & \multirow[t]{2}{*}{$\begin{array}{l}\text { July } \\
\text { Lipiec }\end{array}$} & \begin{tabular}{|l|} 
Daily average air temperature $\left({ }^{\circ} \mathrm{C}\right)$ \\
Średnia dobowa temperatura powietrza $\left({ }^{\circ} \mathrm{C}\right)$
\end{tabular} & 17.7 & 20.5 & 16.4 & 18.2 & \\
\hline & & $\begin{array}{l}\text { Rainfall sum }(\mathrm{mm}) \\
\text { Suma opadów }(\mathrm{mm})\end{array}$ & 38.7 & 24.3 & 69.0 & & 132.0 \\
\hline & \multirow[t]{2}{*}{$\begin{array}{l}\text { August } \\
\text { Sierpień }\end{array}$} & \begin{tabular}{|l|} 
Daily average air temperature $\left({ }^{\circ} \mathrm{C}\right)$ \\
Średnia dobowa temperatura powietrza $\left({ }^{\circ} \mathrm{C}\right)$
\end{tabular} & 19.0 & 19.2 & 21.0 & 19.7 & \\
\hline & & $\begin{array}{l}\text { Rainfall sum }(\mathrm{mm}) \\
\text { Suma opadów }(\mathrm{mm})\end{array}$ & 12.0 & 43.0 & 5.9 & & 60.9 \\
\hline \multirow[t]{10}{*}{2012} & \multirow[t]{2}{*}{$\begin{array}{l}\text { April } \\
\text { Kwiecień }\end{array}$} & $\begin{array}{l}\text { Daily average air temperature }\left({ }^{\circ} \mathrm{C}\right) \\
\text { Średnia dobowa temperatura powietrza }\left({ }^{\circ} \mathrm{C}\right)\end{array}$ & 5.0 & 9.2 & 15.0 & 9.8 & \\
\hline & & $\begin{array}{l}\text { Rainfall sum }(\mathrm{mm}) \\
\text { Suma opadów }(\mathrm{mm})\end{array}$ & 11.5 & 34.4 & 6.9 & & 52.8 \\
\hline & \multirow[t]{2}{*}{$\begin{array}{l}\text { May } \\
\text { Maj }\end{array}$} & \begin{tabular}{|l|} 
Daily average air temperature $\left({ }^{\circ} \mathrm{C}\right)$ \\
Średnia dobowa temperatura powietrza $\left({ }^{\circ} \mathrm{C}\right)$
\end{tabular} & 15.6 & 12.1 & 17.5 & 15.1 & \\
\hline & & $\begin{array}{l}\text { Rainfall sum }(\mathrm{mm}) \\
\text { Suma opadów }(\mathrm{mm})\end{array}$ & 20.1 & 5.8 & 1.0 & & 26.9 \\
\hline & \multirow[t]{2}{*}{$\begin{array}{l}\text { June } \\
\text { Czerwiec }\end{array}$} & $\begin{array}{l}\text { Daily average air temperature }\left({ }^{\circ} \mathrm{C}\right) \\
\text { Średnia dobowa temperatura powietrza }\left({ }^{\circ} \mathrm{C}\right)\end{array}$ & 15.1 & 17.6 & 19.4 & 17.4 & \\
\hline & & $\begin{array}{l}\text { Rainfall sum }(\mathrm{mm}) \\
\text { Suma opadów }(\mathrm{mm})\end{array}$ & 38.7 & 35.3 & 6.0 & & 80.0 \\
\hline & \multirow[t]{2}{*}{$\begin{array}{l}\text { July } \\
\text { Lipiec }\end{array}$} & $\begin{array}{l}\text { Daily average air temperature }\left({ }^{\circ} \mathrm{C}\right) \\
\text { Średnia dobowa temperatura powietrza }\left({ }^{\circ} \mathrm{C}\right)\end{array}$ & 23.5 & 17.0 & 20.1 & 20.2 & \\
\hline & & $\begin{array}{l}\text { Rainfall sum }(\mathrm{mm}) \\
\text { Suma opadów }(\mathrm{mm})\end{array}$ & 54.4 & 60.9 & 3.7 & & 119.0 \\
\hline & \multirow[t]{2}{*}{$\begin{array}{l}\text { August } \\
\text { Sierpień }\end{array}$} & \begin{tabular}{|l|} 
Daily average air temperature $\left({ }^{\circ} \mathrm{C}\right)$ \\
Średnia dobowa temperatura powietrza $\left({ }^{\circ} \mathrm{C}\right)$
\end{tabular} & 21.1 & 16.9 & 19.1 & 19.0 & \\
\hline & & $\begin{array}{l}\text { Rainfall sum }(\mathrm{mm}) \\
\text { Suma opadów }(\mathrm{mm})\end{array}$ & 6.2 & 24.6 & 22.4 & & 53.2 \\
\hline
\end{tabular}


Drzewiecki, S., Pietryga, J., Bereś, P. K. (2016). The effects of chemical control used against males of Diabrotica virgifera LeConte in maize in southern Poland in 2011-2012. Nauka Przyr. Technol., 10, 1, \#7. DOI: 10.17306/J. NPT.2016.1.7

Table 3. Effects of chemical control of Diabrotica virgifera males in 2011 (mean number of beetles in trap)

Tabela 3. Efekty chemicznego zwalczania samców Diabrotica virgifera w 2011 roku (średnia liczba chrząszczy w pułapce)

\begin{tabular}{|c|c|c|c|c|c|c|c|c|c|}
\hline \multirow[b]{2}{*}{$\begin{array}{l}\text { No. } \\
\text { Lp. }\end{array}$} & \multirow{2}{*}{$\begin{array}{c}\text { Application date, experi- } \\
\text { mental setup } \\
\text { Termin zastosowania, } \\
\text { kombinacja } \\
\text { doświadczalna }\end{array}$} & \multirow{2}{*}{$\begin{array}{c}\text { Before } \\
\text { applica- } \\
\text { tion } \\
\text { Przed } \\
\text { zabiegiem }\end{array}$} & \multicolumn{3}{|c|}{$\begin{array}{l}\text { After application } \mathrm{T} 1 \\
\text { Po zabiegu } \mathrm{T} 1\end{array}$} & \multicolumn{4}{|c|}{$\begin{array}{l}\text { After applications T1 and T2 } \\
\text { Po zabiegach T1 i T2 }\end{array}$} \\
\hline & & & $\begin{array}{l}1 \text { day } \\
1 \text { dzień }\end{array}$ & $\begin{array}{l}4 \text { days } \\
4 \text { dni }\end{array}$ & $\begin{array}{c}7 \text { days } \\
7 \text { dni }\end{array}$ & $\begin{array}{c}8 \text { days (T1), } \\
1 \text { day (T2) } \\
8 \text { dni (T1), } \\
1 \text { dzień (T2) }\end{array}$ & \begin{tabular}{|c}
11 days (T1), \\
4 days (T2) \\
11 dni (T1), \\
4 dni (T2)
\end{tabular} & $\begin{array}{c}14 \text { days (T1) } \\
7 \text { days (T2) } \\
14 \text { dni (T1) } \\
7 \text { dni (T2) }\end{array}$ & $\begin{array}{l}21 \text { days (T1), } \\
14 \text { days (T2) } \\
21 \text { dni (T1), } \\
14 \text { dni (T2) }\end{array}$ \\
\hline 1. & Control - Kontrola & $36.8^{\mathrm{a}}$ & $31.3^{\mathrm{a}}$ & $36.3^{\mathrm{a}}$ & $28.8^{\mathrm{a}}$ & $25.8^{\mathrm{a}}$ & $20.8^{\mathrm{a}}$ & $15.3^{\mathrm{a}}$ & $13.3^{\mathrm{a}}$ \\
\hline 2. & T1: Proteus 110 OD & $34.5^{\mathrm{a}}$ & $5.0^{\mathrm{e}}$ & $4.8^{\mathrm{c}}$ & $5.8^{\mathrm{f}}$ & $6.0^{\mathrm{d}-\mathrm{g}}$ & $9.5^{\mathrm{bcd}}$ & $9.8^{\mathrm{a}-\mathrm{d}}$ & $8.0^{\mathrm{a}}$ \\
\hline 3. & T1: Steward $30 \mathrm{WG}$ & $29.0^{\mathrm{a}}$ & $23.3^{\mathrm{ab}}$ & $26.0^{\mathrm{b}}$ & $18.0^{\text {bcd }}$ & $15.8^{\mathrm{b}}$ & $13.8^{\mathrm{b}}$ & $11.5^{\mathrm{a}}$ & $9.5^{\mathrm{a}}$ \\
\hline 4. & T1: Alverde 240 SC & $30.5^{\mathrm{a}}$ & $6.0^{\mathrm{e}}$ & $5.3^{\mathrm{c}}$ & $7.5^{\text {ef }}$ & $7.3^{\mathrm{c}-\mathrm{f}}$ & $11.3^{\mathrm{bc}}$ & $12.8^{\mathrm{a}}$ & $8.0^{\mathrm{a}}$ \\
\hline 5. & T1: Mospilan 20 SP & $33.0^{\mathrm{a}}$ & $7.0^{\mathrm{de}}$ & $4.0^{\mathrm{c}}$ & $10.8^{\mathrm{c}-\mathrm{f}}$ & $9.0^{c-d}$ & $11.3^{\mathrm{bc}}$ & $12.8^{\mathrm{a}}$ & $9.8^{\mathrm{a}}$ \\
\hline 6. & T1: Karate Zeon 050 CS & $26.3^{\mathrm{a}}$ & $1.8^{\mathrm{e}}$ & $2.8^{\mathrm{c}}$ & $9.8^{\text {def }}$ & $11.3^{\mathrm{bc}}$ & $12.0^{\mathrm{b}}$ & $10.5^{\mathrm{ab}}$ & $9.5^{\mathrm{a}}$ \\
\hline 7. & $\begin{array}{l}\text { T1: Alverde } 240 \text { SC } \\
\text { T2: Karate Zeon } 050 \text { CS }\end{array}$ & $28.5^{\mathrm{a}}$ & $3.5^{\mathrm{e}}$ & $4.0^{\mathrm{c}}$ & $7.3^{\mathrm{ef}}$ & $1.5^{\mathrm{gh}}$ & $3.3^{\mathrm{e}}$ & $3.3^{\mathrm{e}}$ & $6.0^{\mathrm{a}}$ \\
\hline 8. & $\begin{array}{l}\text { T1: Alverde } 240 \mathrm{SC} \\
\text { T2: Steward } 30 \mathrm{WG}\end{array}$ & $25.3^{\mathrm{a}}$ & $3.8^{\mathrm{e}}$ & $3.3^{\mathrm{c}}$ & $4.8^{\mathrm{f}}$ & $6.0^{\mathrm{d}-\mathrm{g}}$ & $11.3^{\mathrm{bc}}$ & $10.0^{\mathrm{abc}}$ & $10.8^{\mathrm{a}}$ \\
\hline 9. & $\begin{array}{l}\text { T1: Proteus } 110 \text { OD } \\
\text { T2: Karate Zeon 050 CS }\end{array}$ & $22.8^{\mathrm{a}}$ & $4.3^{\mathrm{e}}$ & $3.5^{\mathrm{c}}$ & $5.3^{\mathrm{f}}$ & $0.8^{\mathrm{h}}$ & $2.3^{\mathrm{e}}$ & $2.8^{\mathrm{e}}$ & $4.3^{\mathrm{a}}$ \\
\hline 10. & $\begin{array}{l}\text { T1: Proteus } 110 \text { OD } \\
\text { T2: Alverde } 240 \text { SC }\end{array}$ & $37.5^{\mathrm{a}}$ & $5.8^{\mathrm{e}}$ & $3.5^{\mathrm{c}}$ & $6.8^{\mathrm{ef}}$ & $2.0^{\mathrm{gh}}$ & $4.3^{\mathrm{de}}$ & $4.3^{\text {cde }}$ & $7.8^{\mathrm{a}}$ \\
\hline 11. & $\begin{array}{l}\text { T1: Mospilan } 20 \text { SP } \\
\text { T2: Alverde } 240 \text { SC }\end{array}$ & $25.3^{\mathrm{a}}$ & $5.5^{\mathrm{e}}$ & $3.8^{\mathrm{c}}$ & $8.8^{\mathrm{ef}}$ & $2.3^{\text {fgh }}$ & $4.3^{\text {de }}$ & $5.3^{\mathrm{b}-\mathrm{e}}$ & $7.0^{\mathrm{a}}$ \\
\hline 12. & $\begin{array}{l}\text { T1: Mospilan } 20 \text { SP } \\
\text { T2: Bi } 58 \text { Nowy } 400 \text { EC }\end{array}$ & $31.0^{\mathrm{a}}$ & $8.3^{\mathrm{de}}$ & $6.0^{\mathrm{c}}$ & $9.8^{\text {def }}$ & $0.0^{\mathrm{h}}$ & $1.8^{\mathrm{e}}$ & $3.3^{\mathrm{e}}$ & $7.3^{\mathrm{a}}$ \\
\hline 13. & $\begin{array}{l}\text { T1: Steward } 30 \mathrm{WG} \\
\text { T2: Mospilan } 20 \mathrm{SP}\end{array}$ & $22.3^{\mathrm{a}}$ & $16.8^{\text {bcd }}$ & $19.8^{\mathrm{b}}$ & $19.3^{b c}$ & $9.0^{\mathrm{cd}}$ & $6.3^{\text {cde }}$ & $5.0^{\mathrm{b}-\mathrm{e}}$ & $6.8^{\mathrm{a}}$ \\
\hline 14. & $\begin{array}{l}\text { T1: Steward } 30 \mathrm{WG} \\
\text { T2: Calypso } 480 \mathrm{SC}\end{array}$ & $26.0^{\mathrm{a}}$ & $21.8^{\mathrm{ab}}$ & $22.3^{\mathrm{b}}$ & $15.8^{\mathrm{b}-\mathrm{e}}$ & $7.5^{\text {cde }}$ & $4.5^{\mathrm{de}}$ & $4.0^{\text {cde }}$ & $6.0^{\mathrm{a}}$ \\
\hline 15. & $\begin{array}{l}\text { T1: Steward } 30 \mathrm{WG} \\
\text { T2: Proteus } 110 \mathrm{OD}\end{array}$ & $28.0^{\mathrm{a}}$ & $18.5^{\mathrm{bc}}$ & $18.0^{\mathrm{b}}$ & $20.0^{\mathrm{ab}}$ & $7.3^{\mathrm{c}-\mathrm{f}}$ & $4.3^{\text {de }}$ & $3.8^{\text {de }}$ & $4.8^{\mathrm{a}}$ \\
\hline 16. & $\begin{array}{l}\text { T1: Karate Zeon } 050 \text { CS } \\
\text { T2: Mospilan } 20 \mathrm{SP}\end{array}$ & $25.0^{\mathrm{a}}$ & $3.3^{\mathrm{e}}$ & $4.3^{\mathrm{c}}$ & $5.5^{\mathrm{f}}$ & $3.3^{\mathrm{e}-\mathrm{h}}$ & $3.5^{\mathrm{e}}$ & $4.8^{\mathrm{b}-\mathrm{e}}$ & $8.8^{\mathrm{a}}$ \\
\hline 17. & $\begin{array}{l}\text { T1: Bi } 58 \text { Nowy } 400 \text { EC } \\
\text { T2: Karate Zeon } 050 \text { CS }\end{array}$ & $29.5^{\mathrm{a}}$ & $1.5^{\mathrm{e}}$ & $3.0^{\mathrm{c}}$ & $4.5^{\mathrm{f}}$ & $1.3^{\mathrm{gh}}$ & $2.3^{\mathrm{e}}$ & $4.3^{\text {cde }}$ & $5.8^{\mathrm{a}}$ \\
\hline 18. & $\begin{array}{l}\text { T1: Calypso } 480 \text { SC } \\
\text { T2: Bi } 58 \text { Nowy } 400 \text { EC }\end{array}$ & $31.5^{\mathrm{a}}$ & $9.5^{\text {cde }}$ & $7.8^{\mathrm{c}}$ & $12.8^{\mathrm{b}-\mathrm{f}}$ & $1.5^{\text {gh }}$ & $3.3^{e}$ & $4.0^{\text {cde }}$ & $6.5^{\mathrm{a}}$ \\
\hline \multicolumn{2}{|c|}{$\mathrm{LSD}_{0.05}-\mathrm{NIR}_{0,05}$} & 20.1 & 9.9 & 9.2 & 9.1 & 5.0 & 5.6 & 6.1 & 6.0 \\
\hline
\end{tabular}

T1 - 16 August, T2 - 23 August.

Means in columns followed by the same letter do not differ at $5 \%$ level of significance.

$\mathrm{T} 1$ - 16 sierpnia, T2 - 23 sierpnia.

Średnie w kolumnach oznaczone tą samą literą nie różnią się na poziomie istotności 5\%. 
Drzewiecki, S., Pietryga, J., Bereś, P. K. (2016). The effects of chemical control used against males of Diabrotica virgifera LeConte in maize in southern Poland in 2011-2012. Nauka Przyr. Technol., 10, 1, \#7. DOI: 10.17306/J. NPT.2016.1.7

The second treatment (T2) was carried out after 20 August in selected experimental setups. At that time maize plants were at developmental stage $\mathrm{BBCH} 73$. The second treatment prolonged the insecticidal effect on beetles for another 4-7 days. On the first day after the treatment the highest efficiency in controlling WCR was found for insecticides containing lambda-cyhalothrin and dimethoate. However, differences in the number of beetles between experimental plots treated with lambda-cyhalothrin and dimethoate and those treated with methaflumizon and acetamiprid were not statistically significant. The greatest differences in the insecticidal effects of the analysed products was found on day 4 after treatment T2, when the number of beetles in most experimental setups was 1.8-6.3 per PAL trap. The number of beetles in the experimental plot treated with insecticides containing methaflumizon (T1) and indoxacarb (T2) was comparable to that in experimental setups treated only once. None of the investigated active ingredients showed insecticidal effect on day 14 after the second treatment. In 2011, throughout the whole study period, the weakest insecticidal effect was found for the product containing indoxacarb (Table 3 ).

In 2012 the peak of flights for adult D. virgifera in the study area was also recorded in the second half of August. The first chemical treatment (T1) was carried out on 20 August, when plants were at developmental stage BBCH 75-79. On days 1 and 4 after treatment all investigated active ingredients, but particularly the product containing dimethoate, were highly effective in controlling the number of WCR beetles. A significant reduction in the number of beetles was found in all experimental plots when compared to the control. A good insecticidal effect for most investigated products was also found on day 7 after T1 treatment. The weakest insecticidal effect on WCR beetles was found for the experimental setup sprayed with methaflumizon, where as early as on day 7 after plant treatment the number of beetles was comparable to the control plot (Table 4).

Table 4. Effects of chemical control of Diabrotica virgifera males in 2012 (mean number of beetles in trap)

Tabela 4. Efekty chemicznego zwalczania samców Diabrotica virgifera w 2012 roku (średnia liczba chrząszczy w pułapce)

\begin{tabular}{|c|c|c|c|c|c|c|c|c|c|}
\hline \multirow[b]{2}{*}{$\begin{array}{l}\text { No. } \\
\text { Lp. }\end{array}$} & \multirow{2}{*}{$\begin{array}{c}\text { Application date, experi- } \\
\text { mental setup } \\
\text { Termin zastosowania, } \\
\text { kombinacja } \\
\text { doświadczalna }\end{array}$} & \multirow{2}{*}{$\begin{array}{l}\text { Before } \\
\text { applica- } \\
\text { tion } \\
\text { Przed } \\
\text { zabiegiem }\end{array}$} & \multicolumn{3}{|c|}{$\begin{array}{l}\text { After application T1 } \\
\text { Po zabiegu T1 }\end{array}$} & \multicolumn{4}{|c|}{$\begin{array}{l}\text { After applications } \mathrm{T} 1 \text { and } \mathrm{T} 2 \\
\text { Po zabiegach } \mathrm{T} 1 \mathrm{i} \text { T2 }\end{array}$} \\
\hline & & & $\begin{array}{l}1 \text { day } \\
1 \text { dzień }\end{array}$ & $\begin{array}{l}4 \text { days } \\
4 \text { dni }\end{array}$ & $\begin{array}{c}7 \text { days } \\
7 \text { dni }\end{array}$ & $\begin{array}{c}8 \text { days (T1), } \\
1 \text { day (T2) } \\
8 \text { dni (T1), } \\
1 \text { dzień (T2) }\end{array}$ & $\begin{array}{c}11 \text { days (T1) } \\
4 \text { days (T2) } \\
11 \text { dni (T1) } \\
4 \text { dni (T2) }\end{array}$ & $\begin{array}{c}14 \text { days (T1), } \\
7 \text { days (T2) } \\
14 \text { dni (T1), } \\
7 \text { dni (T2) }\end{array}$ & $\begin{array}{c}21 \text { days (T1), } \\
14 \text { days (T2) } \\
21 \text { dni (T1), } \\
14 \text { dni (T2) }\end{array}$ \\
\hline 1 & 2 & 3 & 4 & 5 & 6 & 7 & 8 & 9 & 10 \\
\hline 1. & Control - Kontrola & $10.3^{\mathrm{a}}$ & $13.0^{\mathrm{a}}$ & $40.3^{\mathrm{a}}$ & $30.3^{\mathrm{a}}$ & $28.0^{\mathrm{a}}$ & $26.8^{\mathrm{a}}$ & $16.8^{\mathrm{ab}}$ & $12.0^{\mathrm{a}}$ \\
\hline 2. & T1: Proteus 110 OD & $11.5^{\mathrm{a}}$ & $3.5^{\mathrm{b}}$ & $7.8^{\mathrm{b}}$ & $10.0^{\mathrm{bc}}$ & $11.3^{\mathrm{bcd}}$ & $13.3^{\mathrm{bc}}$ & $9.3^{\mathrm{bc}}$ & $6.5^{\mathrm{ab}}$ \\
\hline 3. & T1: Steward $30 \mathrm{WG}$ & $9.0^{\mathrm{a}}$ & $3.3^{\mathrm{b}}$ & $8.0^{\mathrm{b}}$ & $9.5^{\mathrm{bc}}$ & $12.0^{\mathrm{bcd}}$ & $16.3^{\mathrm{b}}$ & $11.0^{\mathrm{bc}}$ & $7.3^{\mathrm{ab}}$ \\
\hline 4. & T1: Alverde 240 SC & $9.5^{\mathrm{a}}$ & $4.0^{\mathrm{b}}$ & $15.8^{\mathrm{b}}$ & $23.8^{\mathrm{a}}$ & $24.5^{\mathrm{a}}$ & $25.0^{\mathrm{a}}$ & $19.5^{\mathrm{a}}$ & $11.5^{\mathrm{a}}$ \\
\hline 5. & T1: Mospilan 20 SP & $10.0^{\mathrm{a}}$ & $3.8^{\mathrm{b}}$ & $6.3^{\mathrm{b}}$ & $10.0^{\mathrm{bc}}$ & $13.0^{\mathrm{b}}$ & $18.0^{\mathrm{b}}$ & $14.5^{\mathrm{abc}}$ & $9.0^{\mathrm{ab}}$ \\
\hline 6. & T1: Karate Zeon 050 CS & $12.0^{\mathrm{a}}$ & $3.0^{\mathrm{b}}$ & $6.8^{\mathrm{b}}$ & $12.3^{\mathrm{bc}}$ & $12.8^{\mathrm{bc}}$ & $17.8^{\mathrm{b}}$ & $14.5^{\mathrm{abc}}$ & $7.3^{\mathrm{ab}}$ \\
\hline
\end{tabular}


Drzewiecki, S., Pietryga, J., Bereś, P. K. (2016). The effects of chemical control used against males of Diabrotica virgifera LeConte in maize in southern Poland in 2011-2012. Nauka Przyr. Technol., 10, 1, \#7. DOI: 10.17306/J. NPT.2016.1.7

Table 4 - cont. / Tabela 4 - cd.

\begin{tabular}{|c|c|c|c|c|c|c|c|c|c|}
\hline 1 & 2 & 3 & 4 & 5 & 6 & 7 & 8 & 9 & 10 \\
\hline 7. & $\begin{array}{l}\text { T1: Alverde } 240 \text { SC } \\
\text { T2: Karate Zeon } 050 \text { CS }\end{array}$ & $9.3^{\mathrm{a}}$ & $4.8^{\mathrm{b}}$ & $16.5^{\mathrm{b}}$ & $22.3^{\mathrm{ab}}$ & $4.5^{\mathrm{b}-\mathrm{e}}$ & $7.5^{\text {cde }}$ & $10.0^{\mathrm{bc}}$ & $6.3^{\mathrm{ab}}$ \\
\hline 8. & $\begin{array}{l}\text { T1: Alverde } 240 \mathrm{SC} \\
\text { T2: Steward } 30 \mathrm{WG}\end{array}$ & $7.5^{\mathrm{a}}$ & $3.5^{\mathrm{b}}$ & $16.3^{\mathrm{b}}$ & $25.5^{\mathrm{a}}$ & $6.5^{\mathrm{b}-\mathrm{e}}$ & $7.8^{\text {cde }}$ & $10.5^{\mathrm{bc}}$ & $8.3^{\mathrm{ab}}$ \\
\hline 9. & $\begin{array}{l}\text { T1: Proteus } 110 \text { OD } \\
\text { T2: Karate Zeon 050 CS }\end{array}$ & $10.8^{\mathrm{a}}$ & $3.3^{\mathrm{b}}$ & $5.5^{\mathrm{b}}$ & $11.0^{\mathrm{bc}}$ & $2.0^{\mathrm{e}}$ & $4.0^{\mathrm{de}}$ & $8.0^{\mathrm{bc}}$ & $5.8^{\mathrm{ab}}$ \\
\hline 10. & $\begin{array}{l}\text { T1: Proteus } 110 \text { OD } \\
\text { T2: Alverde } 240 \text { SC }\end{array}$ & $7.8^{\mathrm{a}}$ & $2.8^{\mathrm{b}}$ & $8.0^{\mathrm{b}}$ & $9.0^{\mathrm{bc}}$ & $5.0^{\mathrm{b}-\mathrm{e}}$ & $11.8^{\mathrm{bcd}}$ & $12.3^{\mathrm{abc}}$ & $10.5^{\mathrm{ab}}$ \\
\hline 11. & $\begin{array}{l}\text { T1: Mospilan } 20 \text { SP } \\
\text { T2: Alverde } 240 \text { SC }\end{array}$ & $9.5^{\mathrm{a}}$ & $4.0^{\mathrm{b}}$ & $7.3^{\mathrm{b}}$ & $10.3^{\mathrm{bc}}$ & $7.3^{\mathrm{b}-\mathrm{e}}$ & $13.5^{\mathrm{bc}}$ & $14.8^{\mathrm{abc}}$ & $10.3^{\mathrm{ab}}$ \\
\hline 12. & $\begin{array}{l}\text { T1: Mospilan } 20 \text { SP } \\
\text { T2: Bi } 58 \text { Nowy } 400 \text { EC }\end{array}$ & $9.3^{\mathrm{a}}$ & $4.5^{\mathrm{b}}$ & $7.8^{\mathrm{b}}$ & $11.3^{\mathrm{bc}}$ & $1.0^{\mathrm{e}}$ & $2.8^{\mathrm{e}}$ & $5.3^{\mathrm{c}}$ & $4.8^{\mathrm{ab}}$ \\
\hline 13. & $\begin{array}{l}\text { T1: Steward } 30 \mathrm{WG} \\
\text { T2: Mospilan } 20 \mathrm{SP}\end{array}$ & $9.8^{\mathrm{a}}$ & $2.8^{\mathrm{b}}$ & $4.5^{\mathrm{b}}$ & $7.5^{\mathrm{c}}$ & $5.3^{\mathrm{b}-\mathrm{e}}$ & $6.8^{\text {cde }}$ & $8.8^{\mathrm{bc}}$ & $6.3^{\mathrm{ab}}$ \\
\hline 14. & $\begin{array}{l}\text { T1: Steward } 30 \mathrm{WG} \\
\text { T2: Calypso } 480 \mathrm{SC}\end{array}$ & $10.3^{\mathrm{a}}$ & $3.0^{\mathrm{b}}$ & $6.0^{\mathrm{b}}$ & $9.5^{\mathrm{bc}}$ & $4.0^{\text {cde }}$ & $7.5^{\text {cde }}$ & $7.0^{\mathrm{c}}$ & $6.8^{\mathrm{ab}}$ \\
\hline 15. & $\begin{array}{l}\text { T1: Steward } 30 \mathrm{WG} \\
\text { T2: Proteus } 110 \mathrm{OD}\end{array}$ & $10.8^{\mathrm{a}}$ & $4.0^{\mathrm{b}}$ & $7.8^{\mathrm{b}}$ & $12.0^{\mathrm{bc}}$ & $3.3^{\mathrm{de}}$ & $5.8^{\text {cde }}$ & $8.3^{\mathrm{bc}}$ & $5.5^{\mathrm{ab}}$ \\
\hline 16. & $\begin{array}{l}\text { T1: Karate Zeon } 050 \text { CS } \\
\text { T2: Mospilan } 20 \mathrm{SP}\end{array}$ & $10.3^{\mathrm{a}}$ & $3.0^{\mathrm{b}}$ & $3.3^{\mathrm{b}}$ & $10.0^{\mathrm{bc}}$ & $5.8^{\mathrm{b}-\mathrm{e}}$ & $7.8^{\text {cde }}$ & $10.5^{\mathrm{bc}}$ & $7.8^{\mathrm{ab}}$ \\
\hline 17. & $\begin{array}{l}\text { T1: Bi } 58 \text { Nowy } 400 \text { EC } \\
\text { T2: Karate Zeon } 050 \text { CS }\end{array}$ & $10.0^{\mathrm{a}}$ & $1.0^{\mathrm{b}}$ & $1.5^{\mathrm{b}}$ & $5.5^{\mathrm{c}}$ & $0.5^{\mathrm{e}}$ & $3.0^{\mathrm{de}}$ & $5.3^{\mathrm{c}}$ & $2.8^{\mathrm{b}}$ \\
\hline 18. & $\begin{array}{l}\text { T1: Calypso } 480 \text { SC } \\
\text { T2: Bi } 58 \text { Nowy } 400 \text { EC }\end{array}$ & $12.5^{\mathrm{a}}$ & $4.8^{\mathrm{b}}$ & $10.0^{\mathrm{b}}$ & $13.5^{\mathrm{bc}}$ & $2.0^{\mathrm{e}}$ & $4.0^{\mathrm{de}}$ & $9.0^{\mathrm{bc}}$ & $5.0^{\mathrm{ab}}$ \\
\hline \multicolumn{2}{|c|}{$\mathrm{LSD}_{0.05}-\mathrm{NIR}_{0,05}$} & 9.0 & 4.0 & 9.4 & 7.7 & 5.3 & 5.2 & 5.2 & 4.3 \\
\hline
\end{tabular}

T1 - 20 August, T2 - 28 August.

Means in columns followed by the same letter do not differ at $5 \%$ level of significance.

T1 - 20 sierpnia, T2 - 28 sierpnia.

Średnie w kolumnach oznaczone tą samą literą nie różnią się na poziomie istotności 5\%.

The second chemical treatment (T2) was carried out in late August, when maize plants were at developmental stage BBCH 79-83. The most rapid insecticidal effect was produced by lambda-cyhalothrin, dimethoate and thiacloprid with deltamethrin, which on the first day after T2 treatment reduced on average the number of insects to $0.5-3.3$ beetles per trap. The strong insecticidal effect of these active ingredients lasted up to day 7 after treatment. Other active ingredients (acetamiprid, indoxacarb and thiacloprid) also effectively controlled the population of pest. Regardless of the treatment date the weakest insecticidal effect was produced by methaflumizon. The number of beetles in this experimental setup on day 7 after the second treatment of plants was comparable to that in the control. In 2012 a statistically significant reduction in the number of WCR beetles lasting to day 14 after the second plant treatment was only observed after the application of the insecticide containing lambda-cyhalothrin (Table 4). 
Drzewiecki, S., Pietryga, J., Bereś, P. K. (2016). The effects of chemical control used against males of Diabrotica virgifera LeConte in maize in southern Poland in 2011-2012. Nauka Przyr. Technol., 10, 1, \#7. DOI: 10.17306/J. NPT.2016.1.7

\section{Discussion}

Sivčev et al. (2012) reported that adult $D$. virgifera pose a hazard to maize only when they occur in high numbers on plants shedding pollen. In Serbia, the population peak for WCR beetles usually occurs after pollen shedding, but mass infestations at the end of July and in early August may damage cob silk and cause loss in grain yield.

Van Rozen and Ester (2010) reported two main reasons for the control of WCR beetles, i.e. to prevent damage to cobs, and to reduce the number of females. This was also indicated by Ciobanu et al. (2011/A).

In Poland there are currently four insecticides approved for the control of adult D. virgifera, and they contain two active ingredients: indoxacarb and thiacloprid with deltamethrin. Other active ingredients tested in this study (methaflumizon, acetamiprid, dimethoate, thiacloprid and lambda-cyhalothrin) have been approved in Poland for the control of related pest species from the family Chrysomelidae - Colorado potato beetle (Leptinotarsa decemlineata Say) and cereal leaf beetle (Oulema spp.).

The study demonstrated that each of the tested active ingredients produced stronger or weaker insecticidal effects on the population of $D$. virgifera beetles. These findings, indicating the usefulness of the tested active ingredients in controlling adult $D$. virgifera, support the results from previous studies by Bereś (2013), who investigated the same active ingredients under the soil and climate conditions of south-eastern Poland on a five- and six-year long maize crop monoculture. The previous study demonstrated the strongest insecticidal effect produced by thiacloprid with deltamethrin, methaflumizon, lambda-cyhalothrin, dimethoate and thiacloprid. Slightly lower efficiency was found for products containing acetamiprid and indoxacarb. Similar results were obtained in this study. However, weaker insecticidal effects on WCR beetles were produced by indoxacarb in 2011 and methaflumizon in 2012, which was not observed in previous studies by Bereś (2013).

Considerable usefulness of selected active ingredients from the group of synthetic pyretroids and chloronicotinils for the control of $D$. virgifera beetles was reported by Ciobanu et al. (2011/A), who confirmed in their experiments the strong insecticidal effects of cypermethrin, lambda-cyhalothrin, deltamethrin, zeta-cypermethrin and thiacloprid.

Some active ingredients used in this study (dimethoate, lambda-cyhalothrin, thiacloprid, acetamiprid and deltamethrin) are used for the control of WCR beetles in Hungary and Pennsylvania (van Rozen and Ester, 2006).

The study demonstrated that in a several-year-long maize crop monoculture the insecticidal effect of tested products used in a single treatment (T1) was maintained for 4-7 days after treatment. A second spraying (T2) with a different active ingredients prolonged the effects of chemical control for another 4-7 days, and in only one case (lambda-cyhalothrin) for 14 days.

The study suggests that maize grown for several years in a monoculture is at greater risk of mass infestation by WCR beetles and should be treated twice to control the pest. This was also indicated earlier by Bereś and Drzewiecki (2011), who recorded short-term insecticidal effects on WCR beetles after a single treatment of maize with products containing thiacloprid, thiacloprid + deltamethrin, lambda-cyhalothrin, dimethoate, acetamiprid, and chlorpyriphos + cypermethrin. 
Drzewiecki, S., Pietryga, J., Bereś, P. K. (2016). The effects of chemical control used against males of Diabrotica virgifera LeConte in maize in southern Poland in 2011-2012. Nauka Przyr. Technol., 10, 1, \#7. DOI: 10.17306/J. NPT.2016.1.7

The study also revealed differences in optimum dates for the control of $D$. virgifera beetles between regions of Poland. In south-eastern Poland the optimum date for the control of WCR beetles usually falls in the first half of August (Bereś, 2013; Bereś and Sionek, 2010b), but in this study it was the second half of August, which is in line with previous findings by Drzewiecki and Pietryga (2009) for southern Poland.

\section{Conclusions}

1. In the study years the optimum time for the control of $D$. virgifera males in southern Poland was in the second half of August.

2. A strong insecticidal effect on WCR beetles was found for products containing acetamiprid, dimethoate, indoxacarb, lambda-cyhalothrin, methaflumizon, thiacloprid and thiacloprid with deltamethrin.

3. The best pest control was achieved by using two treatments on maize plants, and insecticides containing active ingredients from different chemical groups.

4. On a maize field grown in a several-year-long monoculture a single spraying with insecticide was insufficient to control the population of $D$. virgifera beetles.

\section{References}

Adamczewski, K., Matysiak, K. (2011). Kukurydza. In: Klucz do określania faz rozwojowych roślin jedno- i dwuliściennych w skali BBCH (pp. 27-30). Poznań: Instytut Ochrony Roślin.

Bača, F. (1994). New member of the harmful entomofauna of Yugoslavia Diabrotica virgifera virgifera LeConte (Coleoptera, Chrysomelidae). Zašt. Bilja, 208, 45, 2, 125-131.

Baufeld, P., Enzian, S. (2005). Western corn rootworm (Diabrotica virgifera virgifera), its potential spread and economic and ecological consequences in Germany. In: D. V., Alford, G. F., Backhouse (eds.), Plant protection and plant health in Europe: introduction and spread of invasive species (pp. 149-154). Berlin: Humboldt University.

Bereś, P. K. (2013). Studium nad doskonaleniem integrowanej ochrony kukurydzy przed zachodnią kukurydzianą stonką korzeniową (Diabrotica virgifera virgifera LeConte) i omacnicą prosowianką (Ostrinia nubilalis Hbn.). Rozpr. Nauk. Inst. Ochr. Rośl., 29.

Bereś, P. K., Drzewiecki, S. (2011). Przydatność wybranych insektycydów do zwalczania chrząszczy Diabrotica virgifera LeConte w kukurydzy (Zea mays L.). Prog. Plant Prot. / Post. Ochr. Rośl., 51, 1, 167-176.

Bereś, P. K., Sionek, R. (2010a). Study on the fecundity, egg collection technique and longevity of Diabrotica virgifera LeConte. J. Plant Prot. Res., 50, 4, 429-437.

Bereś, P. K., Sionek, R. (2010b). The occurrence and sex ratio of Diabrotica virgifera LeConte beetles on sweet maize (Zea mays var. saccharata) in south-eastern Poland near Rzeszów in 2007-2009. Veg. Crops Res. Bull., 73, 87-97.

Ciobanu, C., Ciobanu, G., Sandor, M., Domuta, C., Albu, R., Popov, C., Domuta, C., Vuscan, A. (2011/A). Research on the establishment of chemical control technology on adults of species Diabrotica virgifera virgifera LeConte (Western corn rootworm). An. Univ. Oradea Fasc. Ecotoxicol. Zooteh. Tehnol. Ind. Aliment., 10/A, 10, 83-90. 
Drzewiecki, S., Pietryga, J., Bereś, P. K. (2016). The effects of chemical control used against males of Diabrotica virgifera LeConte in maize in southern Poland in 2011-2012. Nauka Przyr. Technol., 10, 1, \#7. DOI: 10.17306/J. NPT.2016.1.7

Ciosi, M., Miller, N. J., Kim, K. S., Giordano, R., Estoup, A., Guillemaud, T. (2008). Invasion of Europe by the western corn rootworm, Diabrotica virgifera virgifera: multiple transatlantic introductions with various reductions of genetic diversity. Mol. Ecol., 17, 16, 3614-3627.

Decyzja wykonawcza Komisji z dnia 6 lutego 2014 r. uchylająca decyzję 2003/766/WE w sprawie środków nadzwyczajnych zapobiegających rozprzestrzenianiu się we Wspólnocie Diabrotica virgifera LeConte. (2014). Dz. Urz. UE, L, 38, 45.

Drzewiecki, S., Pietryga, J. (2009). Obserwacje nad występowaniem zachodniej kukurydzianej stonki korzeniowej (Diabrotica virgifera LeConte) w południowo-zachodnim rejonie Polski. Prog. Plant Prot. / Post. Ochr. Rośl., 49, 4, 1681-1685.

Dyrektywa Parlamentu Europejskiego i Rady 2009/128/WE z dnia 21 października 2009 r. ustanawiająca ramy wspólnotowego działania na rzecz zrównoważonego stosowania pestycydów. (2009). Dz. Urz. UE, L, 309, 71-86.

EPPO. (2012). Present situation (2011) of Diabrotica virgifera virgifera in Europe. Obtained from: http://www.eppo.int/QUARANTINE/special_topics/Diabrotica_virgifera/diabrotica_virgifera.htm

Kiss, J., Komáromi, J., Bayar, K., Edwards, C. R., Hatala-Zsellér, I. (2005). Western corn rootworm (Diabrotica virgifera virgifera LeConte) and the crop rotation systems in Europe. In: S. Vidal, U. Kuhlmann, C.R. Edwards (eds.), Western corn rootworm: ecology and management (pp. 189-220). Wallingford: CABI Publishing.

Meinke, L. J. (1995). Adult corn rootworm management. Univ. Nebr. Agric. Res. Div. Misc. Publ., 63.

Metcalf, R. L. (1986). Forward. In: J. L. Krysan, T. A. Miller (eds.), Methods for the study of pest Diabrotica (pp. 7-15). New York: Springer.

Pruess, K. P., Witkowski, J. F., Raun, E. S. (1974). Population suppression of western corn rootworm by adult control with ULV malathion. J. Econ. Entomol., 67, 651-655.

van Rozen, K., Easter, A. (2006). Chemical control against Diabrotica v. virgifera LeConte: a review of the historical and current pest control strategies. Report Diabr-Act. EU Funded Project FP6-2004-SSP-4-022623. Paris: Arvalis - Institut du végétal.

van Rozen, K., Easter, A. (2010). Chemical control of Diabrotica virgifera virgifera LeConte. J. Appl. Entomol., 134, 5, 376-384.

Rozporządzenie Ministra Rolnictwa i Rozwoju Wsi z dnia 24 kwietnia 2014 r. uchylające rozporządzenie w sprawie zwalczania i zapobiegania rozprzestrzenianiu się zachodniej kukurydzianej stonki korzeniowej. (2014). Dz. U., item 707.

Sahajdak, A., Bereś, P. K., Konefał, T. (2006). Diabrotica virgifera LeConte - a new threat to maize crops in Poland and measures taken against the pest. J. Plant Prot. Res., 46, 2, 157-161.

Sivčev, I., Kljajić, P., Kosić, M., Sivčev, L., Stanković, S. (2012). Management of western corn rootworm (Diabrotica virgifera virgifera). Pestic. Phytomed., 27, 3, 189-201.

Sivčev, I., Manojlović, B., Krnjajić, S., Dimić, N., Draganić, M., Bača, F., Kaitović, Ž., Sekulić, R., Kereši, T. (1994). Distribution and harmfulness of Diabrotica virgifera LeConte (Coleoptera, Chrysomelidae), a new maize pest in Yugoslavia. Zašt. Bilja, 208, 45, 1, 19-26.

Tuska, T., Kiss, J., Edwards, R. C. (2002). Establishing economic thresholds for feeding by western corn rootworm adults in commercial corn. In: Book of abstracts. Plant Protection Society of Serbia, $9^{\text {th }}$ IWGO Diabrotica Subgroup Meeting and $8^{\text {th }}$ EPPO ad-hoc Panel, Belgrade, 3-5 November (p. 50). 
Drzewiecki, S., Pietryga, J., Bereś, P. K. (2016). The effects of chemical control used against males of Diabrotica virgifera LeConte in maize in southern Poland in 2011-2012. Nauka Przyr. Technol., 10, 1, \#7. DOI: 10.17306/J. NPT.2016.1.7

\section{EFEKTY CHEMICZNEGO ZWALCZANIA \\ SAMCÓW DIABROTICA VIRGIFERA LeCONTE \\ NA KUKURYDZY W POŁUDNIOWEJ POLSCE W LATACH 2011-2012}

Streszczenie. Badania wykonano w południowej Polsce w miejscowości Sławików w latach 2011-2012 na polu kukurydzy prowadzonym w monokulturze. Do zwalczania chrząszczy Diabrotica virgifera wykorzystano insektycydy zawierające następujące substancje czynne: acetamipryd, dimetoat, indoksakarb, lambda-cyhalotryna, metaflumizon, tiachlopryd oraz tiachlopryd + deltametryna. Optymalny termin zwalczania chrząszczy ustalono za pomocą pułapek feromonowych typu PAL firmy Csalomon ${ }^{\circledR}$. Badane insektycydy stosowano w formie jedno- i dwukrotnego opryskiwania roślin w okresie licznego występowania chrząszczy, który przypadał w drugiej połowie sierpnia. Stwierdzono, że wszystkie badane substancje czynne pozwalały skutecznie ograniczać liczebność owadów zwykle do 7 dni od zabiegu opryskiwania roślin. Wykonanie drugiego zabiegu chemicznego pozwalało skutecznie zmniejszyć liczebność populacji szkodnika w kolejnych 7 dniach.

Słowa kluczowe: stonka kukurydziana, chrząszcze, kukurydza, insektycydy, substancja czynna

Corresponding address - Adres do korespondencji:

Sławomir Drzewiecki, Oddziat w Sośnicowicach, Instytut Ochrony Roślin - Państwowy Instytut Badawczy, ul. Gliwicka 29,44-153 Sośnicowice, Poland, e-mail: s.drzewiecki@ior.gliwice.pl

Accepted for publication - Zaakceptowano do opublikowania:

26.10.2015

For citation - Do cytowania:

Drzewiecki, S., Pietryga, J., Bereś, P. K. (2016). The effects of chemical control used against males of Diabrotica virgifera LeConte in maize in southern Poland in 2011-2012. Nauka Przyr. Technol., 10, 1, \#7. DOI: 10.17306/J.NPT.2016.1.7 